\title{
Psychiatry: time for change
}

\section{Donatella Marazziti and Liliana Dell'Osso}

Dipartimento di Medicina Clinica e Sperimentale, Section of Psychiatry, University of Pisa, Pisa, Italy

First published online 10 September 2014

Psychiatry has changed. The terrific increase in neuroscientific research, which has occurred in the recent past few decades, has permitted the spread of the notion that the mind corresponds in part to the brain, and that psychiatric symptoms are also the results of disturbances of the brain. ${ }^{1}$ In addition, it has provided us sharpened instruments, ie, psychotropic drugs that, far from being specific, at least are not "big bullets," as they used to be in the beginning of the psychopharmacological era. Moreover, neuroscientific research has opened (and will open increasingly) new horizons to more focused psychiatric interventions and, therefore, has reduced the stigma related to mental disorders, as well as to decrease the gap between common people and psychiatry. $^{2}$ Therefore, we can affirm that we know much about the pathophysiology of mental disorders, and that we can control most psychiatric disorders and symptoms, and, in some cases, we can even restore normal equilibrium by means of chemical compounds.

Besides drugs, psychological practice has also become more scientifically oriented, with some techniques showing reliable effectiveness, so that the combination of drugs and focused psychological treatment can be extremely beneficial in some patients. ${ }^{3}$ This is also possible because diagnostic instruments have become more appropriate, widely applied, and constitute a sort of common language which undergoes continuous refinements, on the basis of the feedback arriving from the bedside and the eventual support of biological correlates. The link between research and clinical practice has undoubtedly become stronger in psychiatry than in any other medical field.

However, it is likely that the equation brain + mind is not so absolute: are we really sure that the brain will be able to accomplish the goal of understanding itself, and therefore to reveal the mystery of its self-consciousness? Are we sure that when we have dissected all psychiatric

* Address for correspondence: Donatella Marazziti, MD, Dipartimento di Medicina Clinica e Sperimentale Section of Psychiatry, University of Pisa, Via Roma 67, Pisa 56100, Italy.

(Email: dmarazzi@psico.med.unipi.it) disorders into distinct symptoms or symptom clusters, and we have understood all their molecular and/or neurological bases or genetic underpinnings, will we have then reached a crucial point in psychiatry? Although it is true that the mind is rooted in the brain, and that the understanding of the brain in molecular terms will promote great advancements, it is also plausible that the mind is something more complex and will escape even our current attempts at definition or delimitation. ${ }^{4}$ There is a shared impression that if we proceed in this way, that is to say, toward progressive dissections of complex phenomena, such as psychiatric symptoms, we shall never arrive at the end. Different concepts have been introduced to overcome this bias, such as comorbidity, dimensions, subthreshold phenomena, and psychopathological continuum, to the extent that even the schizophrenia subtypes are not considered reliable. ${ }^{5,6}$ Indeed, at this point, we need to incorporate all data deriving from neuroscientific research (which cannot be neglected nowadays even by most traditionally oriented psychoanalysts), in more exhaustive and comprehensive models, including the challenges deriving from the environment, which, through learning and/or epigenetic mechanisms, has had a profound effect on brain development. If this is true for almost all branches of medicine, there can be little doubt that psychiatry especially is a discipline that is linked to and a reflection of social urges, demands, problems, and fears, since the individual may be considered to be the result of a constant interaction between the brain and its environment. For this reason, when applied in the diagnosis, treatment, and, when successful, resolution of specific disturbances or disorders, psychiatrists restore not only a deranged brain equilibrium, but, in addition, an individual's attitudes and behaviors and, consequently, the effects that a single individual may have upon society. It may equally be concluded that the particular society or environment by which an individual is surrounded, together with any conflicts that may exist therein, can affect the functioning of that individual and induce mental disorders or, at the very least, act as a triggering 
factor in those more vulnerable members of that society. It is therefore important that psychiatry (as well as medicine generally) be sufficiently flexible to take into account constant inputs deriving from a rapidly changing world, which can affect or even create hitherto unknown disorders. For example, the onset of Internet addiction can be related to the widespread use of new technologies (PC and smartphone), the increase in pathological gambling to the availability of gambling opportunities, ${ }^{7}$ and posttraumatic stress disorder (PTSD) to novel threats. ${ }^{8}$

We must, therefore, look for other ways of thinking about the brain/mind, its functioning, and disturbances. Besides that, we must have the courage to propose hypotheses beyond the currently accepted paradigms, because scientific advancements occur in a linear way, on the basis of investment and energy devoted to them, but also through breaking points. This means that we should be also more creative and start to build up new hypotheses, especially at this point that data continue to be accumulated at a speed sometimes surpassing our possibility of processing them. The current neuroscientific paradigms have shown all their weaknesses when transferred to the therapeutic side. If accomplishments have been enormous and have permitted the management of psychiatric disorders with better tolerability profiles, a large percentage of patients still fail to respond. This is not surprising if we consider that most of the drugs were discovered by serendipity, and that most of hypotheses are drug-based and constitute the bases for the developments of new compounds (the drug as tool paradigm). In addition, as the pressure to manage severe psychiatric disorders is decreasing, there is an apparent lesser need of novel drugs, so that pharmaceutical companies and government agencies invest less in research, which is also motivated by current economic constraints. However, it is essential that scientists continue to address and pressure politicians to promote investment in CNS research and not to decrease budgets in this area. In the U.S., President Obama called for an initiative aimed at deepening our understanding of the brain, ${ }^{9}$ and there is a similar one promoted by the European Community. ${ }^{10}$ It is worth mentioning that about 20 years ago, the so-called "brain decade" fostered several neuroscientists and led to intriguing achievements in CNS research. ${ }^{11}$

A starting point for the emergence of new ideas might be the awareness that is time for psychiatry to change again, and to be reshaped by taking into account and including the entire range of approaches. It is time for psychiatry to integrate in a comprehensive manner biological hypotheses and pharmacological treatment with psychotherapy and psychosocial constructs, ${ }^{12}$ while keeping in mind that the complexity of a single patient cannot be described solely by ICD or DSM diagnostic criteria. $^{3,13}$

It can be easily predicted that there will be an increasing demand for psychiatric interventions in the future (depression will be the second cause of disability in 2020) that, for this reason, should be more scientifically based, more ethical, and probably accustomed to different cultural contexts, especially those wherein the access to mental health services is still meager. This implies a greater flexibility of all psychiatric operatives and operations that must be ready to accept, approach, and possibly resolve the inputs and challenges coming from a changing world. "Integration" and "flexibility" should be the main features of the future psychiatry. Psychiatry has changed, but it needs to change again and constantly.

\section{Disclosures}

The authors do not have anything to disclose.

\section{REFERENCES:}

1. Andreasen N. What is psychiatry? Am J Psychiatry 1997; 154(5): 591-593.

2. Corrigan PW, Watson AC. Understanding the impact of stigma on people with mental illness. World Psychiatry 2002; 1(1): 16-20.

3. Stahl SM. Psychotherapy as an epigenetic 'drug': psychiatric therapeutic target symptoms linked to malfunctioning brain circuits with psychotherapy as well as with drugs. J Clin Pharm Ther 2012; 37(3): 249-253.

4. Edelman G, Tononi G. A Universe of Consciousness: How Matter Becomes Imagination. New York: Basic Books; 2000.

5. van Os J. The dynamics of subthreshold psychopathology: implications for diagnosis and treatment. Am J Psychiatry 2013; 170 (7): 695-698.

6. Dell'Osso L, Pini S. What did we learn from research on comorbidity in psychiatry? Advantages and limitations in the forthcoming DSMV era. Clin Pract Epidemiol Ment Health 2012; 8: 180-184.

7. Marazziti D, Presta S, Baroni S, Silvestri S, Dell'Osso L. Behavioral addictions: a novel challenge for psychopharmacology. CNS Spectr In press. DOI: http://dx.doi.org/10.1017/S1092852913001041.

8. Shalev AY, Freedman S. PTSD following terrorist attacks: a prospective evaluation. Am J Psychiatry 2005; 162(6): 1188-1191.

9. Advisory Committee to the Director. Brain Research through Advancing Innovative Neurotechnologies (BRAIN) Working Group. Bethesda, MD: National Institutes of Health; 2013.

10. Alivisatos AP, Chun M, Church GM, Greenspan RJ, Roukes ML, Yuste R. The brain activity map project and the challenge of functional connectomics. Neuron 2012; 74(6): 970-974.

11. Jones EG, Mendell LM. Assessing the decade of the brain. Science 1999; 284(5415): 739-743.

12. Thase ME. Psychopharmacology in conjunction with psychotherapy. In: Snyder CR, Ingram RE, eds. Handbook of Psychological Change: Psychotherapy Process and Practices for the 21st Century. New York: John Wiley \& Sons; 2000: 474-497.

13. Reis de Oliveira I, Schwartz T, Stahl SM. Integrating Psychotherapy and Psychopharmacology. London: Routledge; 2014. 